\title{
Optical properties of daylight curable resin doped with nanodiamond powder
}

\author{
Krzysztof Królewski, Aleksandra Wieloszyńska, Aleksandra Kamińska, Katarzyna Kardacz ${ }^{1}$ \\ Department of Metrology and Optoelectronics, Faculty of Electronics, Telecommunications and Informatics, \\ Gdańsk University of Technology, Narutowicza Street 11/12, 80-233 Gdańsk, Poland
}

Received September 04, 2019; accepted September 27, 2019; published September 30, 2019

\begin{abstract}
In this paper a new material for 3D printing was elaborated on. Since diamond has very good optical properties, an idea occurred to us to apply it in a $3 \mathrm{D}$ printing process. A mixture of nanodiamond powder and standard 3D printing resin was created and several printouts have been completed. They have been tested for their abilities to transmit and absorb light in a wide spectrum of wavelengths. It turned out that nanopowder doped resin in comparison to standard one has worse optical properties. However, it shows that a mixture of resin and nanopowder can control optical properties of printouts.
\end{abstract}

$3 \mathrm{D}$ printing is a very promising technology and it is used in many applications, e.g. rapid prototyping, production of spare parts [1-3]. Depending on the used technique, many types of this technology can be mentioned. One of them is a digital light processing method using a DLP projector and light-curable resin [3]. The method is characterized by high speed of printing and good mechanical parameters of printouts [4-5].

In our experiment a new material for 3D printing has been prepared and analyzed. This material consists of standard polymer resin and diamond nanoparticles. Such a combination gives interesting optical properties, which were tested in this study. As a standard polymer resin, we have applied amber 3D Daylight Hard resin (Photocentric), which is widely available and used in 3D printing. The used printer was Liquid Crystal 10' 3D printer (Photocentric).

As an example of printouts some prototypes of cylindrical and spherical lenses have been printed (Fig. 1 and Fig. 2).

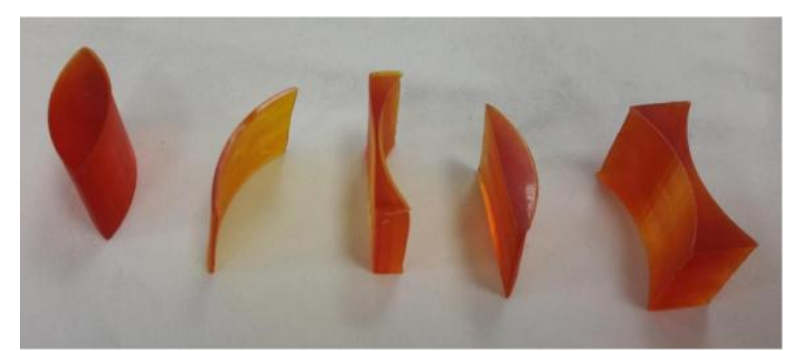

Fig. 1. Prototypes of cylindrical lenses which were printed using a 3D printer. From the left: convexo-convex lens, convexo-concave lens, plano-concave lens, plano-convex lens, concavo-concave lens.

\footnotetext{
${ }^{1}$ E-mail: katarzyna.kardacz@pg.edu.pl
}

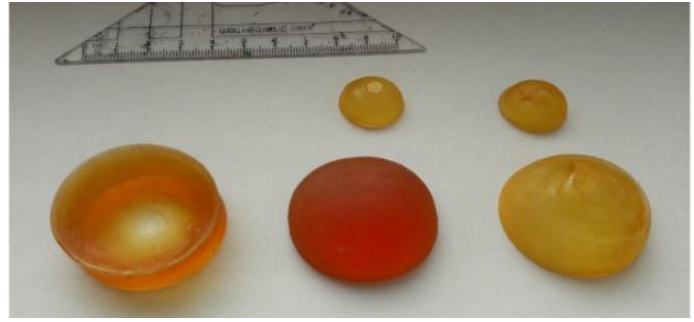

Fig. 2. Prototypes of printed spherical lenses. From the bottom left: plano-concave lens, plano-convex lens, and convex-concave lens. In the upper part of the photo, there are two convex-concave lenses.

To compare the results, the optical properties of the used standard resin was tested as well. The $20 \mathrm{~mm} \times$ $20 \mathrm{~mm}$ plates were printed as test samples which were prepared with different thickness $(0.2 \mathrm{~mm}, 0.5 \mathrm{~mm}$, $0.8 \mathrm{~mm}, 1 \mathrm{~mm}, 1.5 \mathrm{~mm}, 2 \mathrm{~mm}$ and $5 \mathrm{~mm}$ ). The two series of printouts are shown in Fig 3.

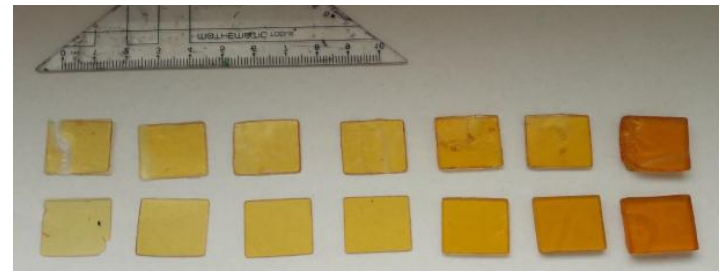

Fig. 3. Two series of flat plates (first series at the top and a second at the bottom). The thinnest plate is on the left.

The transmission characteristics of manufactured printouts were obtained on the Metash UV-9000 spectrophotometer. The experiment was carried out at room temperature and in the wavelength range $200 \mathrm{~nm}$ $1100 \mathrm{~nm}$. The transmission characteristics for both series of printing are characterized by increasing transmission with decreasing the thickness of plates. The characteristics are introduced in Fig. 4 and Fig. 6.

The transmission for the second series is higher than the transmission for the first series. This is especially noticeable for thicker plates. In the first series, the maximal transmission is $60 \%$ and $44 \%$ for $2 \mathrm{~mm}$ and $5 \mathrm{~mm}$ thick plate, respectively. In turn, in the second series, this value is $75 \%$ and $65 \%$, respectively. 


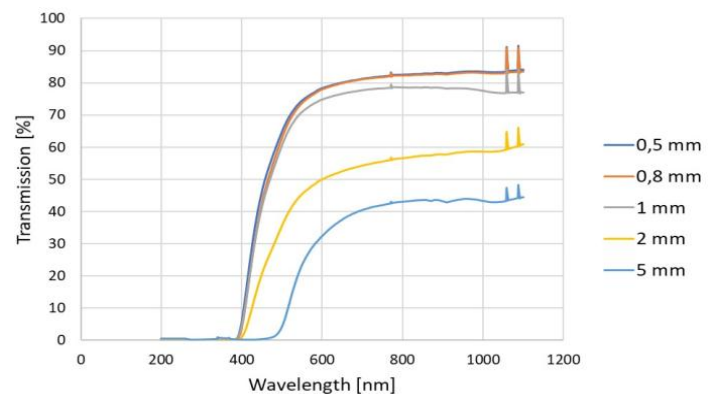

Fig. 4. Transmission characteristics for the first series of flat plates. The transmission is almost zero for light waves in the range of $200 \div 400$ $\mathrm{nm}$. The greatest transmission is for light waves in the range of $800 \div 1100 \mathrm{~nm}$.

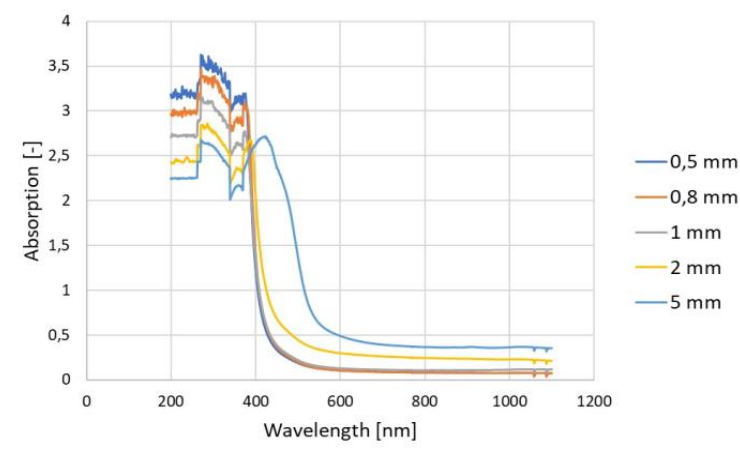

Fig. 5. Absorption characteristics the first series of flat plates. The greatest absorption is for light waves in the range of $200 \div 400 \mathrm{~nm}$. The lowest absorption is for light waves in the range of $600 \div 1100 \mathrm{~nm}$.

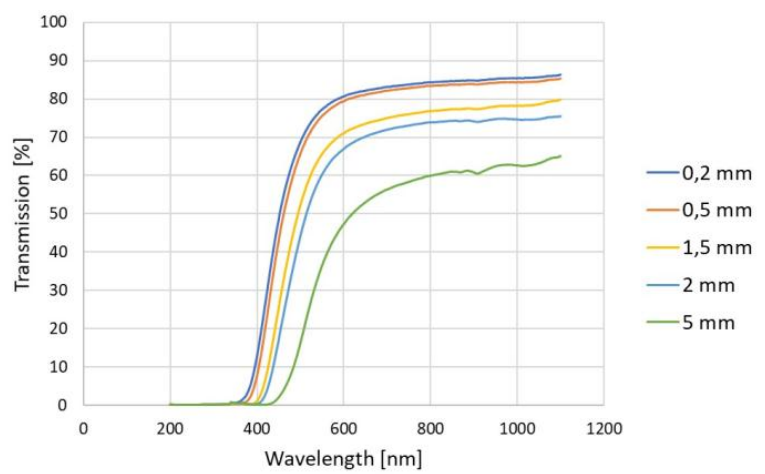

Fig. 6. Transmission characteristics for the second series of flat plates.

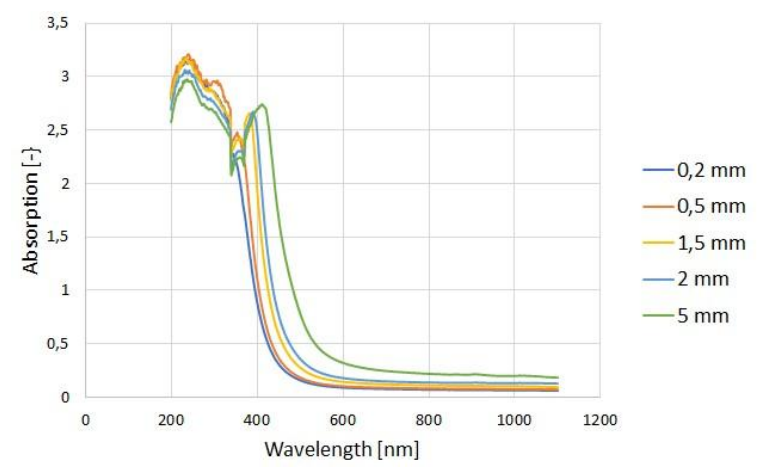

Fig.7. Absorption characteristics for the second series of flat plates.
The obtained transmission characteristics are comparable with transmission characteristics of wellknown optical materials (like N-BK7, fused silica) [6-7]. In the case of the proposed material, the transmission characteristics are constant in the range from $600 \mathrm{~nm}$ to $1200 \mathrm{~nm}$, which is appropriate to many optical applications.

The absorption characteristics are introduced in Fig. 5 and Fig. 7. The highest absorbance occurs in the range from 200 to $400 \mathrm{~nm}$. It is expected because of the colour of plates - orange.

To increase application possibilities of manufactured printouts a novel print material has been figured out [8-9]. Next, plates were printed from our new mixture which consists of the standard resin and nanodiamond [10]. The nanodiamond powder was obtained by evaporating DMSO (dimethyl sulfoxide) from the suspension with nanodiamond. Then the $66.835 \mathrm{~g}$ liquid resin was mixed with $0.069 \mathrm{~g}$ powder. First, the magnetic stirring was carried out for an hour and after that, the sonication was done for 45 minutes. The sonicator worked in pulse mode with power set at $10 \%$. One series of printouts is shown in Fig. 8.

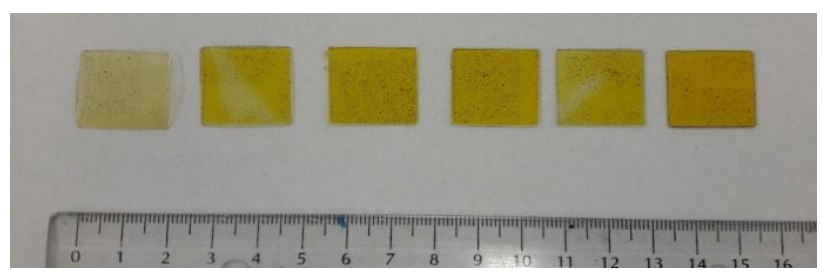

Fig. 8. One series of flat plates The plates were printed from the mixture of resin and nanodiamond powder which was obtained by evaporating DMSO from the suspension with nanodiamond.

For evaluation the material properties of manufactured printouts (homogeneity, distribution of nanoparticles) the series of optical coherence tomography (OCT) measurements have been carried out. The results are presented in Fig. 9 and Fig. 10.

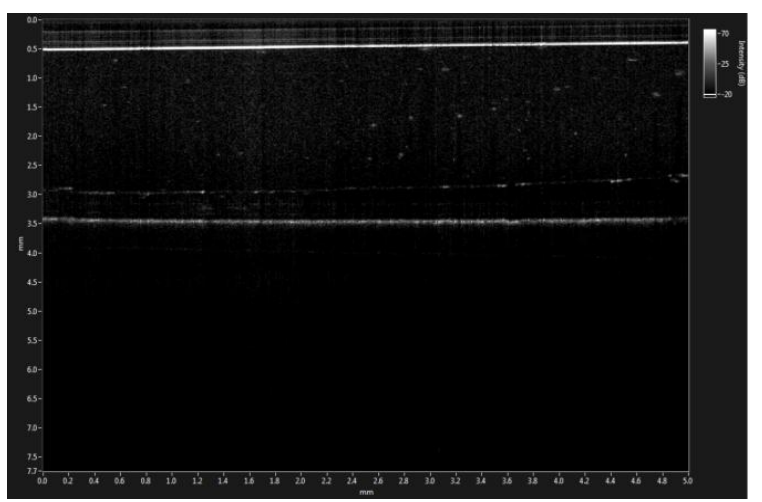

Fig. 9. OCT image of the plate with nanopowder. There are scattering centres in the sample (bright spots in the image), which indicates the occurrence of diamond nanoparticles. 


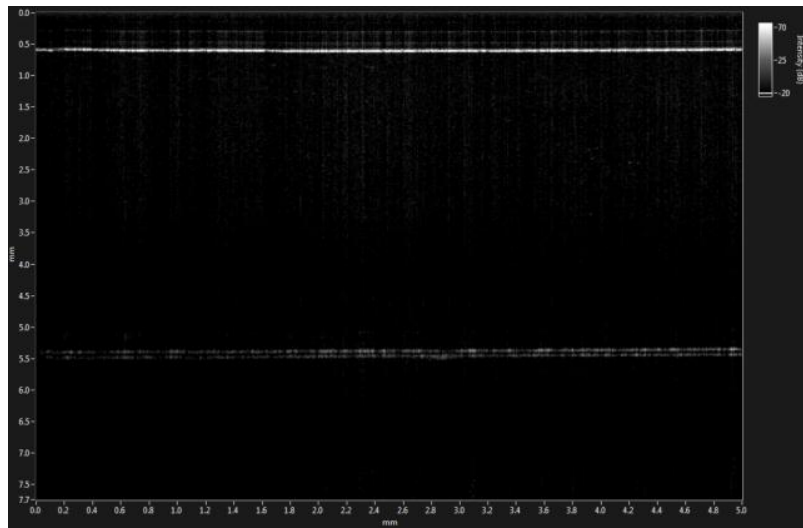

Fig. 10. OCT image of polymer used for printing. Lack of the scattering centres indicates no occurrence of the diamond nanoparticles.

Single B-scans have been obtained from three plates with diamond nanoparticles and one plate without nanoparticles as the reference. Basing on OCT images it can be noticed that the prepared material was homogeneous due to the presence of nanoparticles (they occur in the entire volume) and due to the lack of tendency to agglomeration.

Transmission characteristics and absorption characteristics for the flat plates which were printed from the resin with nanodiamond are introduced in Fig. 11 and Fig. 12, respectively.

The highest values of transmission were observed in the range of $600 \div 1100 \mathrm{~nm}$. The maximal transmission is $86 \%$ and around $80 \%$ for $0.2 \mathrm{~mm}$ and $0.8 \mathrm{~mm}$ thick plates, respectively. In the range of $190 \div 400 \mathrm{~nm}$ the transmission is almost equal to zero. In the range of $400 \div 600 \mathrm{~nm}$ there is a switch between low and high values of transmission. This switch is not so smooth as for the plates without nanodiamond powder (Fig. 4 and Fig. 6).

There is a similar trend in absorption characteristics in Fig. 12. In the range of $400 \div 600 \mathrm{~nm}$ there is a switch between high and low values of absorption.

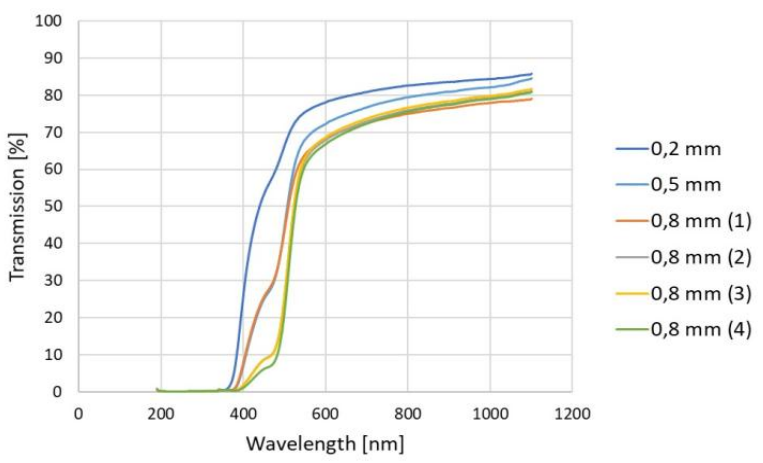

Fig. 11. Transmission characteristics for the flat plates which were created from the mixture of resin and nanodiamond powder. In the range of $400-600 \mathrm{~nm}$ there is a switch between low and high values of transmission.

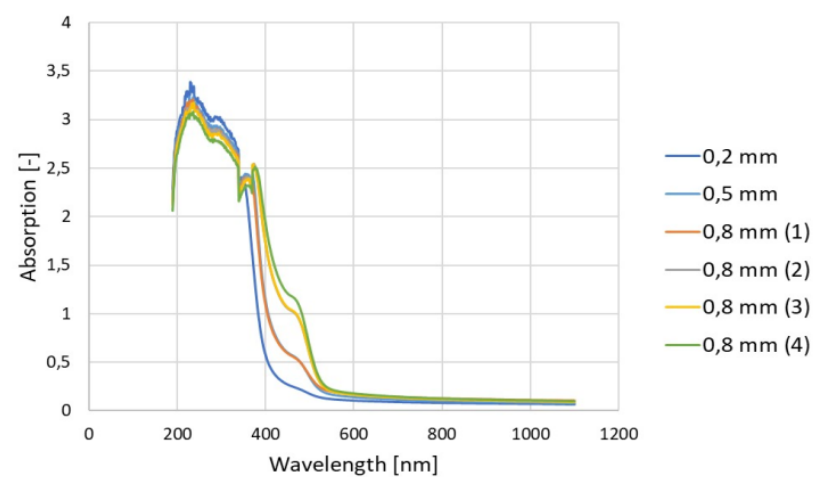

Fig. 12. Absorption characteristics for the flat plates which were created from the mixture of resin and nanodiamond powder. In the range of $400 \div 600 \mathrm{~nm}$ there is a switch between high and low values of absorption.

To conclude, we have proven that it is possible to fabricate a new material for 3D printing, which is a mixture of standard polymer resin and diamond nanoparticles. In this research we have shown that the presence of nanodiamond affects the optical characteristics of the mixture. It gives premises that other nanoparticles can modify the optical properties, especially absorption characteristics. Therefore, it may lead to new opportunities for a low-cost, quick and easy method for rapid prototyping of optical filters.

\section{References}

[1] L. Ding, R. Wei, H. Che, Proc. Engin. 85, 123 (2014).

[2] L. Fang, T. Chen, R. Li, S. Liu, IEEE Sensors J. 16(17), 6604 (2016).

[3] G.B. Kim, S. Lee, H. Kim, D.H. Yang, Y.H. Kim, Y.S. Kyung et al., Korean J. Radiology, 17(2), 182 (2016).

[4] J.W. Stansbury, M.J. Idacavage, Dental Materials, 32(1), 54 (2016).

[5] G.H. Wu, S.H. Hsu, J. Med. Biol. Eng. 35(3), 285-292 (2015)

[6] https://www.thorlabs.com/newgrouppage9.cfm?objectgroup_id=69 $73 \&$ tabname $=\mathrm{N}-\mathrm{BK} 7$

[7] https://www.thorlabs.com/images/TabImages/UVFS_Transmission _780.gif

[8] U. Kalsoom, A. Peristyy, P.N. Nesterenko, B. Paull, RSC Advances, 6(44), 38140 (2016).

[9] K.M. El-Say, J. Appl. Pharmac. Science 01(06), 29 (2011).

[10] K. Królewski, MSc thesis, 3D printing of optical elements from diamond powders (2018). 